\title{
Topographical Guidance of PSC-Derived Cortical Neurons
}

\author{
Joke Terryn $\mathbb{D}^{1,2,3}$ Marleen Welkenhuysen, ${ }^{4}$ Olga Krylychkina, ${ }^{4}$ Andrea Firrincieli, ${ }^{4}$ \\ Alexandru Andrei, ${ }^{4}$ Veerle Reumers, ${ }^{4}$ Philip Van Damme, ${ }^{2,3,5}$ Dries Braeken, ${ }^{4}$ \\ and Catherine Verfaillie ${ }_{(\mathbb{D}}{ }^{1}$
}

\author{
${ }^{1}$ Interdepartmental Stem Cell Institute, Department of Development and Regeneration, KU Leuven, 3000 Leuven, Belgium \\ ${ }^{2}$ Laboratory of Neurobiology, Center for Brain \& Disease Research, VIB, 3000 Leuven, Belgium \\ ${ }^{3}$ Department of Neurology, University Hospitals Leuven, 3000 Leuven, Belgium \\ ${ }^{4}$ Department of Life Science Technologies, IMEC, 3001 Leuven, Belgium \\ ${ }^{5}$ Experimental Neurology, Department of Neuroscience, KU Leuven, 3000 Leuven, Belgium
}

Correspondence should be addressed to Catherine Verfaillie; catverfaillie@icloud.com

Received 18 May 2018; Accepted 30 August 2018; Published 18 October 2018

Academic Editor: Elisa Castagnola

Copyright (C) 2018 Joke Terryn et al. This is an open access article distributed under the Creative Commons Attribution License, which permits unrestricted use, distribution, and reproduction in any medium, provided the original work is properly cited.

\begin{abstract}
Background and Aims. Human pluripotent stem cell- (PSC-) derived neurons are increasingly used in the study of neurodevelopmental and neurodegenerative diseases. Electrophysiological activity is a crucial and defining function of these neurons. Patch clamping and the more recently developed multielectrode arrays are used to evaluate this complex function. In this study, we assess the effect of topography on PSC-derived neurons as a baseline for the development of next-generation silicon oxide substrates (in direct relation to MEA fabrication) that integrate topography as a means to guide network formation and stimulate differentiation. Methods. Human PSC was differentiated towards cortical neurons and seeded on a silicon micropatterned substrate to screen for topographic guidance and improved neurite outgrowth. We next developed customized micropillar and microgroove substrates to further assess the effect of topography on human cortical neurons. Immunocytochemistry and morphological analyses were used to track neuron differentiation and guidance. Results. Microgroove and micropillar substrates appeared to support the differentiation of human stem cell-derived cortical neurons, promoted neurite outgrowth, and facilitated contact guidance. In contrast to flat surfaces, topography also appeared to limit the number of NESTIN positive neuronal progenitor cells present in the culture. Conclusion. We describe the topographical preferences of human PSC-derived cortical neurons, on neurite outgrowth and guidance. Embedding topography in silicon substrates may be an effective approach to direct and improve neuronal network formation. We propose to integrate topography in multielectrode arrays as a practical method to shape the neuro-electronic interface.
\end{abstract}

\section{Introduction}

Human pluripotent stem cell (hPSC) technology has created unprecedented possibilities in our ability to study human neuronal cells in vitro. Especially the discovery by the Yamanaka group in 2006, that somatic cells could be reprogrammed to pluripotent cells, allows for the facile generation of patient-derived stem cells $[1,2]$. These human-induced pluripotent stem cells (hiPSCs) are increasingly used to model neurodevelopmental and neurodegenerative diseases and hold the promise to enable the discovery of new pharmaceutical and therapeutic targets. Electrophysiological activity, which can be assessed by patch clamping or multielectrode arrays, is a crucial and defining function of neurons. Unfortunately, hPSC-derived neuronal cells require on average more than two months of in vitro culturing before functional synapses are formed $[3,4]$, and even then, they still display an immature electrical profile, resembling an embryonic/early postnatal stage of development [5]. Patch clamping is considered the gold standard for electrophysiological evaluation but is labor-intensive and has low throughput. Multielectrode arrays allow the parallel examination of multiple neurons and their network activity. Currently, the MEA arsenal is evolving from conventional MEAs, combining a standard 
culture dish with electrodes, to more complex MEAs. For example, Lewandowska et al. combined a high-density electrode array with a poly(dimethylsiloxane) channel device, which could isolate axons from the neuronal soma [6]. Another approach to improve MEA microfabrication would be to create a substrate that integrates topography that can guide network formation and can simultaneously stimulate neuronal maturation. A number of studies have demonstrated the ability of topography to direct cell growth, control cell shape and polarity, and affect differentiation and proliferation [7-9]. In general, the response to topography depends on the substrate and the specific surface features that are being introduced and even more on the cell type under examination. The behavior of hPSC-derived neuronal progeny on topographical surfaces is less well described. A small number of studies demonstrated the ability of nanotopography to facilitate neuronal differentiation; however, these studies all used softer synthetic polymers (poly(dimethylsiloxane), PDMS; polyurethane-acrylate, PUA) [10-12]. Because MEA electrodes have to be constructed with conductive materials (a silicon oxide substrate with an electrode material like, e.g., titanium nitrite (TiN)), it is not possible to predict the behavior of hPSC-derived neurons on these conductive materials, based on the studies that used PDMS or PUA.

Therefore, we conducted a topographical study with human PSC-derived cortical neurons on silicon oxide substrates. We first tested the effect of a micropatterned substrate, previously described (Micholt et al. [13]), consisting of 150 areas of micropillars with variable diameter and spacing on the neurite outgrowth and directionality of immature PSC neuronal progeny. Next, we designed customized micropillar and microgroove silicon oxide substrates and assessed the effect of these topographies on immature neuronal PSC progeny, as well as on more mature lower layer cortical neuronal progeny. We focused on guidance and improvement of neurite outgrowth and assessed how topography affects cell number and expression of progenitor and differentiation markers.

\section{Materials and Methods}

2.1. PSC Culture. Five different human pluripotent stem cell (PSC) lines were used. These included the BJ1 iPSC line, generated in-house from BJ fibroblasts (CRL-2522, ATCC, USA) as previously described $[14,15]$; the SBAD2 line, derived by StemBANCC and kindly provided by Prof. Paul Jennings, Insbruck University, as part of the EU-ToxRisk consortium (http://www.eu-toxrisk.eu); the 0028 iPSC line (purchased from Sigma, USA); the ChiPSC6b iPSC line (purchased from Cellartis-Takara, Japan); and the H9 human embryonic stem cell (ESC) line (obtained from WiCell, USA) [16]. The generation of iPSC and use of iPSC and hESC were approved by the Ethics Committee of KU Leuven. All PSCs were cultured on 2\% ESC-qualified Matrigel (Becton Dickinson, USA) coated wells in Essential 8 Flex Medium (Thermo Fisher Scientific, USA) and maintained at $37^{\circ} \mathrm{C}$ and $5 \% \mathrm{CO}_{2}$ in a humidified incubator. Cells were split twice a week with EDTA (Lonza, Switzerland).
2.2. PSC Differentiation towards Cortical Neurons. An adapted version of the protocol described by Shi et al. was used to generate cortical neurons [3]. Briefly, single cell suspensions of $2.5 \times 10^{5}$ PSCs per $\mathrm{cm}^{2}$ were plated in mTESR1 medium (Stemcell Technologies, Canada) containing Revitacell (Thermo Fisher Scientific, USA) and allowed to form a confluent monolayer. Neural induction was started by changing the medium to neural maintenance medium (NMM) with the addition of $10 \mu \mathrm{M}$ SB431542 (Tocris, United Kingdom) and $1 \mu \mathrm{M}$ LDN193189 (Miltenyi Biotec, Germany) for 10 days. Differentiating cultures were purified enzymatically two to four times using $1 \mathrm{mg} / \mathrm{ml}$ Dispase II (Roche, Switzerland). Purified neural progenitor cells (NPCs) were cryobanked at day 33 in vitro (DIV33) in NMM containing $10 \%$ DMSO and thawed in NMM containing $20 \mathrm{ng} / \mathrm{ml} \mathrm{FGF-2} \mathrm{(R \& D} \mathrm{Systems,} \mathrm{USA).} \mathrm{To} \mathrm{allow} \mathrm{dif-}$ ferentiation, NPCs were enzymatically dissociated using Accutase (Sigma, USA) and seeded at a density of 25,000 cells per $\mathrm{cm}^{2}$ on microtopography substrates or at a density of 100,000 cells per $\mathrm{cm}^{2}$ in 96 well microplates (Greiner Bio-One, Austria) for characterization. Culture vessels were coated with $50 \mu \mathrm{g} / \mathrm{ml}$ poly-L-ornithin (Sigma, USA) and $10 \mu \mathrm{g} / \mathrm{ml}$ Laminin (Sigma, USA) prior to cell seeding. NPCs were replated on DIV40 and were left to mature until DIV55 for further characterization. NPCs seeded on micropillar or microgroove substrates were fixed after 24 hours (DIV40+1) or 15 days (DIV40+15).

2.3. RNA Extraction, cDNA Synthesis, and Gene Expression. RNA samples were collected on day 0 , day 40 , and day 55 of the cortical neuron differentiation protocol. Total RNA was purified using the GenElute Mammalian kit (Sigma, USA). cDNA was generated using $1 \mu \mathrm{g}$ of RNA with SuperScript reagents (Invitrogen, USA), and qRT-PCR was performed on a Mastercycler Eppendorf Realplex qPCR with a SYBR Green PCR master mix and primers mix at final concentration of $250 \mathrm{nM}$. Primer sequences are listed in Table S1.

2.4. Fabrication of Topographical Chips. Two types of micropillar substrates were used in this study. The initial screening experiments were conducted on a micropillar substrate as previously described by Micholt et al. [13]. This micropatterned substrate of 8000 by $8000 \mu \mathrm{m}$ consists of 150 areas of hexagonal pillars of variable width $(1-5.6 \mu \mathrm{m})$ and spacing $(0.6-15 \mu \mathrm{m})$ with a constant height of $3 \mu \mathrm{m}$. Each topographical pattern covers a growth area of $400 \mu \mathrm{m}$ by $400 \mu \mathrm{m}$. The second topographical array consisted of a micropillar substrate consisting of square pillars $(1.4 \mu \mathrm{m}$ spacing, $2.35 \mu \mathrm{m}$ width, and $3 \mu \mathrm{m}$ height) and a microgrooved substrate (spacing of $4 \mu \mathrm{m}$, width of $4 \mu \mathrm{m}$, and height of $3 \mu \mathrm{m}$ ) created at IMEC. Each customized micropillar or microgroove pattern covers a large growth area of 6000 by $7300 \mu \mathrm{m}$. The arrays were fabricated in a class 1 clean room environment on standard 8-inch Si substrates. The processing started with a plasma-enhanced chemical vapour deposition (PECVD) step of $3.4 \mu \mathrm{m}$ of $\mathrm{SiO} 2$. Then, I-line photolithography was used to define the pattern, and a reactive-ion etch (RIE) process defines the $3 \mu \mathrm{m}$ tall topography (grooves or pillars) on the 
wafers. The remaining photoresist was removed with a wet strip MS2001 solvent chemistry. Surface contaminants were removed from the diced wafer pieces by UV-ozone treatment (1 hour) followed by a 70\% ethanol wash.

2.5. Immunofluorescent Staining and Microscopy. Cells were fixed with $4 \%$ paraformaldehyde (PFA), permeabilized with PBS containing $0.1 \%$ Triton X-100, and blocked with 5\% donkey serum (Jackson ImmunoResearch Laboratories, USA) at room temperature. Primary antibodies were diluted in DAKO antibody diluent (Agilent, USA) in the appropriate dilutions and incubated overnight at $4^{\circ} \mathrm{C}$. To stain neurons (named TUJ1+ cells afterwards), we used rabbit polyclonal anti- $\beta$ III tubulin $(1: 1000,302302$, Synaptic Systems, Germany), and to stain neuronal progenitors (named NESTIN+ cells), we used mouse monoclonal anti-NESTIN (1:300, 10C2, Eurogentec, Belgium). See Figure S1 in Supplementary Materials for the isotype-controlled immunofluorescent staining of these primary antibodies. Cells were then washed with PBS and incubated with appropriate secondary Alexa Fluor Antibodies (Thermo Fisher Scientific, USA) for $45 \mathrm{~min}$ at room temperature. Nuclei were stained with $4 \mu \mathrm{g} / \mathrm{ml}$ Hoechst 33258 (Sigma, USA). Microtopographical chips were imaged using an LSM 780 laser scanning confocal microscope (Carl Zeiss, Germany). Surface topography was imaged using scanning electron microscopy (Nova NanoSEM 200, FEI).

2.6. Morphological Analysis. Neurite outgrowth and expression of progenitor and differentiation markers were analysed using the semiautomated software MetaMorph (Molecular Devices, USA). The Neurite Outgrowth Application Module was used to segment neurites (based on the TUJ1 image) and define cell nuclei (based on the Hoechst 33258 image). Measurements included total and average neurite outgrowths and cell number. The Cell Scoring Application Module was used to define subpopulations of cells (NESTIN positive versus TUJ1 positive).

2.7. Topographical Guidance. The direction of neurite outgrowth in relation to topographic guidance cues was assessed using the "directionality" plugin in ImageJ (open source software). Input images (TUJ1 images) were first converted to a binary image and smoothened to reduce the effect of image pixilation on the assessment of directionality. The "local gradient orientation method" was selected to analyse directionality, producing an orientation map and frequency histogram per angle $\left(0\right.$ to $\left.180^{\circ}\right)$.

2.8. Statistical Analysis. Differences between multiple groups were analysed using a repeated measurements two-way ANOVA (two grouping variables). $P$ values $<0.05$ (adjusted according to Sidak's multiple comparison test) were considered significant. Data were shown as mean, and error bars represent standard error of mean (SEM). Means were reported with standard deviation as measure of variability. $P$ values were represented by asterisks in the graphs according to the following rating: $P<0.05^{*}, P<0.01^{* *}$, $P<0.001^{* * *}$, and $P<0.0001^{* * *}$. Three different hPSC lines (H9, 0028, and CRL-2522) were tested in the screening experiment using chips containing 150 areas of micropillars. Four different hPSC lines were tested in the second experiment, using newly developed arrays containing flat surfaces, micropillars, and microgrooves (three biological replicates for ChiPSC6b, BJ1, and 0028; two biological replicates for the SBAD2 line). Two DIV40+15 samples (derived from lines CRL-2522 and SBAD2, respectively) were partially detached during culture and were excluded from imaging. Therefore, a total of 11 biological replicates were analysed on DIV $40+1$ and a total of 9 biological replicates on DIV $40+15$.

\section{Results}

3.1. Characterization of iPSC-Derived Cortical Neurons. To fate PSCs to cortical neurons, we used an adapted version of the protocol described by Shi et al. [3]. This protocol uses sequential enzymatic purification steps on PSC committed to the neuroectodermal lineage, to generate pure populations of NPCs. Figure 1(a) illustrates the differentiation protocol. We generated NPCs from four hiPSC lines and one hESC line and allowed them to mature up to day 55 (DIV55) for further characterization.

We performed qRT-PCR to measure transcript levels of pluripotency, NPCs, and lower layer cortical neuron marker genes (Figure 1(b)). A decrease in the pluripotency marker OCT4 was observed and an induction of the NPC marker PAX6 by DIV40, followed by the induction of lower layer cortical neuronal markers (TBR1, CTIP2) on DIV55. Transcript levels for PAX6 remained high throughout the differentiation, indicating, as expected, persistence of NPCs among the differentiating cortical neurons.

3.2. Response of PSC-Derived Cortical Neurons to Topography. Micholt et al. previously examined the response of embryonic mouse hippocampal neurons on micropillar substrates (Figure 2(a)) [13]. This micropatterned substrate was made up of 150 areas of hexagonal pillars of variable width $(1-5.6 \mu \mathrm{m})$ and different pillar configurations (pillar spacing ranging from 0.6 to $15 \mu \mathrm{m}$ ). Pillar height was maintained constant at $3 \mu \mathrm{m}$. To investigate if human PSCderived cortical neurons would respond similarly to topography, we seeded DIV40 neural progenitor cells (NPC) from three human PSC lines (H9, 0028, and CRL-2522) on these micropatterned substrates and assessed neurite outgrowth 24 hours later. To detect the effect of the different pillar configurations, we examined neuronal cells present on all 150 pillar configurations. We quantified the total cell number and determined the relative frequency of TUJ1 positive neuronal cells and NESTIN positive NPCs. Neurites (detected in the TUJ1 stained image) were segmented using the semiautomated software MetaMorph (Molecular Devices, USA). Three technical replicates were analysed per PSC line. The heatmaps in Figure 2(c) represent the averaged data from the three different PSC lines. The number of TUJ1 positive cells was high irrespective of the topography, indicating that the PSC progeny retained their neuronal identity. Slightly higher cell numbers were detected on pillar configurations that closely resemble a flat surface (pillar spacing $>4 \mu \mathrm{m}$ ), 

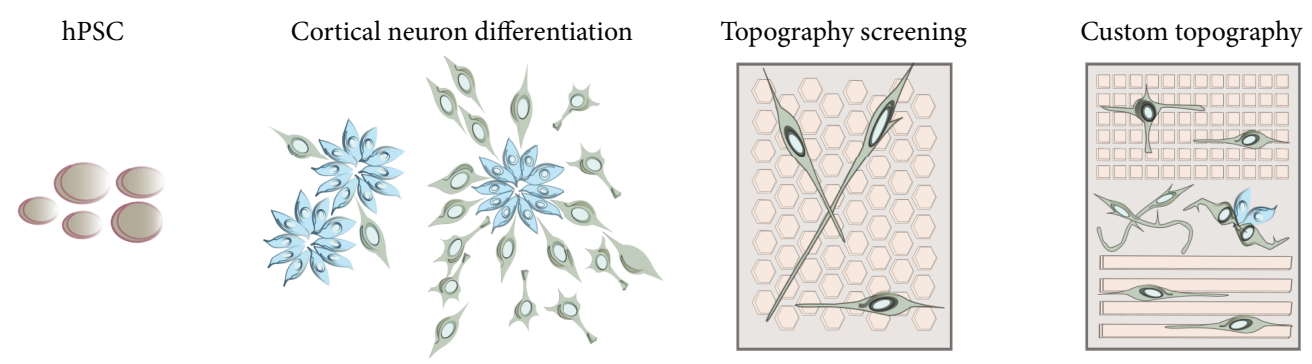

(a)
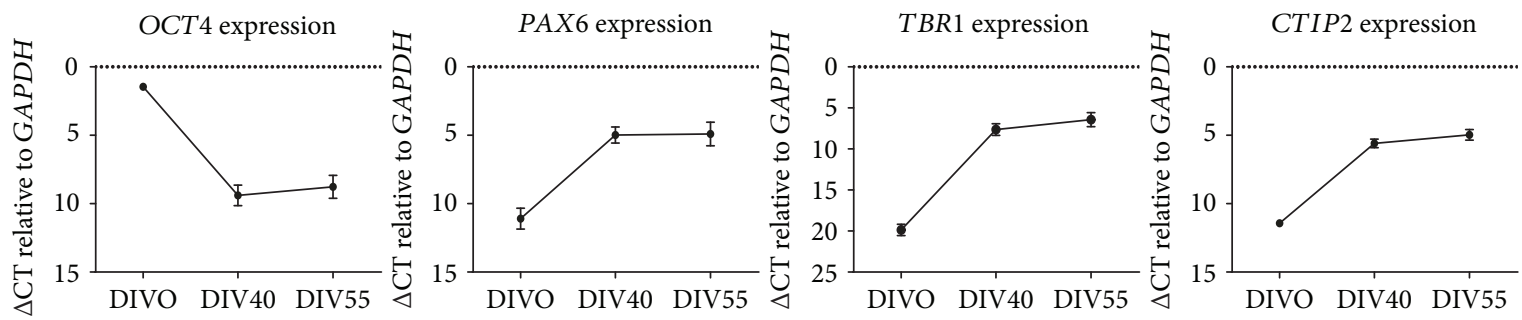

(b)

FIgure 1: Cortical neuron differentiation of human PSCs. (a) Schematic overview of the adapted cortical differentiation protocol by Shi et al. [3]. DIV40 purified cortical neuronal progenitor cells (NPC) were plated on microtopographical substrates. (b) Transcript levels of the pluripotency marker (OCT4), the neural progenitor marker (PAX6), and lower layer cortical neuron markers (TBR1, CTIP2) were assessed at specific time points throughout differentiation for the following PSC lines: the H9 hESC line and the hiPSC lines (0028, CRL-2522, BJ1, and SBAD2). Gene expression was normalized to the expression of the housekeeping gene GAPDH ( $n=2-3$ differentiations per cell line).

and a slightly higher fraction of these cells stained positive for NESTIN. Comparing neurite outgrowth for different pillar dimensions suggested that there was a region of optimal width and spacing that promoted maximal neurite outgrowth with the center focused around pillars spaced 1.4 to $1.6 \mu \mathrm{m}$ apart and featuring diameters of $2.8 \mu \mathrm{m}$. Regions that promoted neurite outgrowth also appeared to induce neurite alignment. Examples of neuronal morphology on different pillar configurations are shown in Figure 2(b).

3.3. Topography Directs Neurite Orientation. Based on these results, as well as on published data on the effect of microgrooves on rodent neuronal behavior in vitro $[13,17]$, we generated new topographical substrates to further examine the effects of topography on human PSC-derived neuronal cultures. We first increased the growth area to enable examination of more neurons. We also changed the pillar shape from hexagonal to square pillars, as this pillar shape will facilitate future electrode design in MEAs. We selected a pillar configuration with pillar spacing of $1.4 \mu \mathrm{m}$, as this spacing promoted maximal neurite outgrowth (Figure 2(c)). For practical reasons, we selected a pillar diameter of $2.35 \mu \mathrm{m}$. We also generated a topographical substrate consisting of grooves. The height of the grooves was identical to the height of the micropillars $(3 \mu \mathrm{m})$. We selected a groove width of $4 \mu \mathrm{m}$ to avoid perpendicular alignment of neurites to the grooves. Grooved substrates most often evoke parallel neurite alignment but can, depending on groove dimensions, elicit perpendicular contact guidance [17]. Figure 3 demonstrates SEM micrographs of the surface topography of the micropillar and microgroove substrates.

To examine the effects of these new topographies on PSC-derived cortical neurons, we seeded DIV40 cortical progenitors from the 0028 and ChiPSC6b iPSC lines (tested previously on the variable pillar substrate), as well as two additional lines, BJ1 and SBAD2, onto flat, pillar, and microgroove substrates. We assessed the cultures 24 hours or 15 days following plating in differentiation medium on the different topographical substrates. Representative micrographs of cells stained with antibodies against the neuronal marker, $\beta 3$-tubulin (TUJ1), and progenitor marker, NESTIN, 15 days after plating (DIV40+15) on the topographical substrates are shown in Figure 4.

Visual inspection of the cultures revealed topography directed neurite outgrowth for all PSC lines. PSC-derived cortical neurons plated on the microgroove substrates aligned parallel to the grooves. By contrast, square micropillars appeared to induce neurite outgrowth of PSC neuronal progeny in two directions $\left(0^{\circ}\right.$ and $\left.90^{\circ}\right)$. To quantify and compare the effect of the different substrates on immature PSC neuronal progeny ( $24 \mathrm{~h}$ after plating) and more mature PSC neuronal progeny (15 days after plating), we analysed the direction of neurite outgrowth (detected in the TUJ1 image) with the directionality plugin of ImageJ (open source software). Without contact guidance, the directionality histogram was nearly flat, representing random growth and an equal distribution of neuronal outgrowth at all angles. The expected frequency per angle for random growth is $0.005\left(1 / 180^{\circ}\right)$. However, pillar and groove topographical cues elicited neurite guidance, with histogram peaks at $90^{\circ}$ for microgroove surfaces and at $0^{\circ}$ and $90^{\circ}$ for micropillar substrates (Figure 5). Examination after $24 \mathrm{~h}$ and 15 days revealed that longer-term culture did not change the shape of the directionality histogram. Moreover, surface microtopography continued to direct neurite outgrowth, even in denser cultures. 


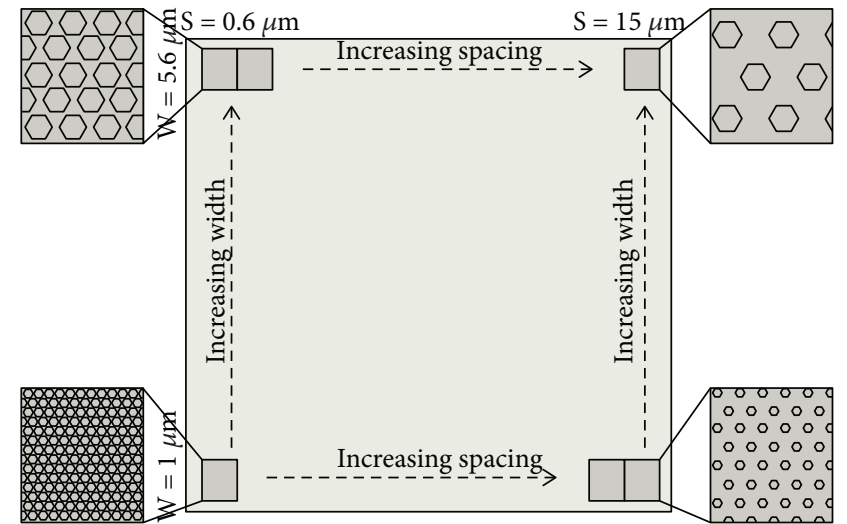

(a)
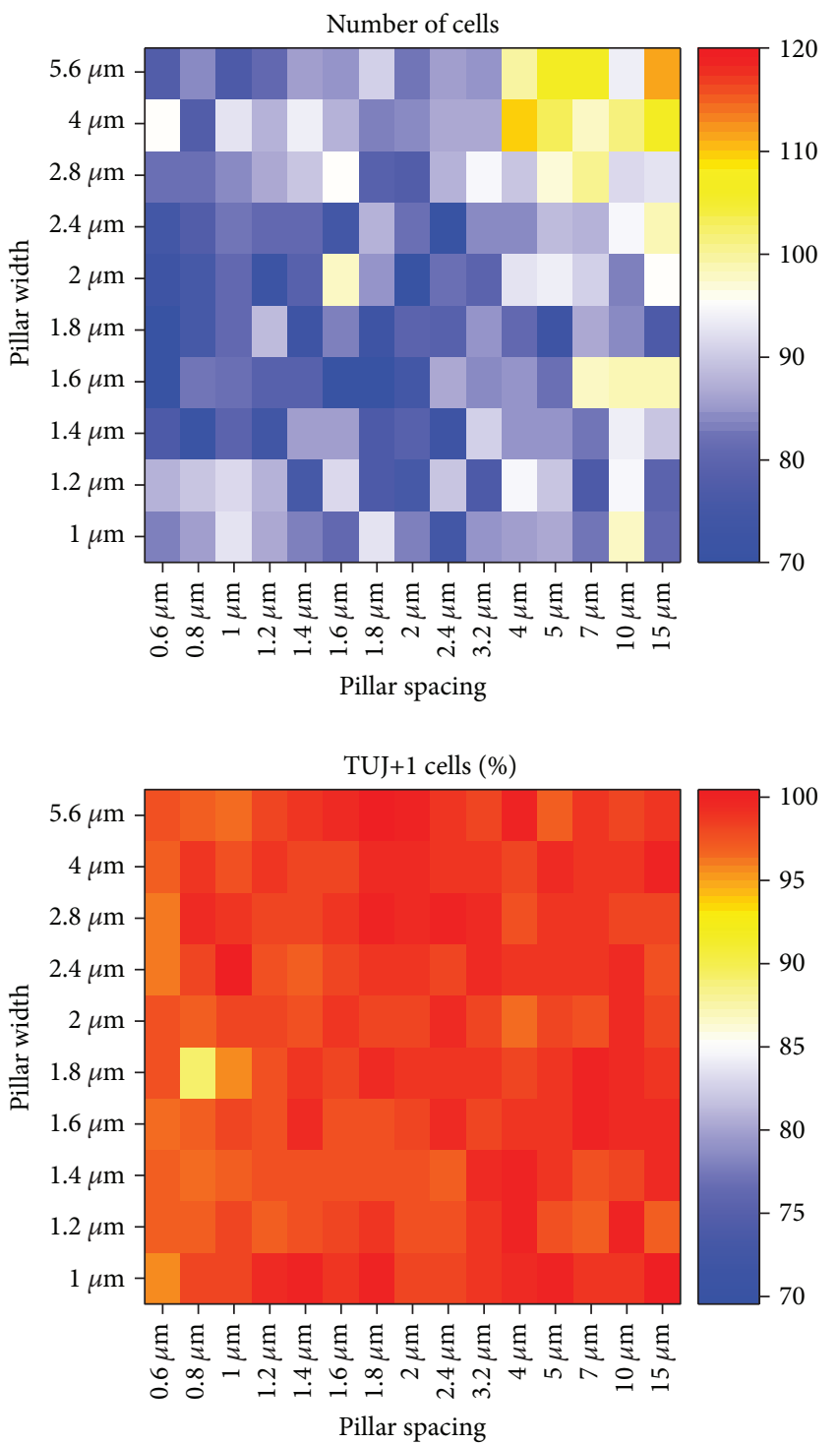

Pillar spacing
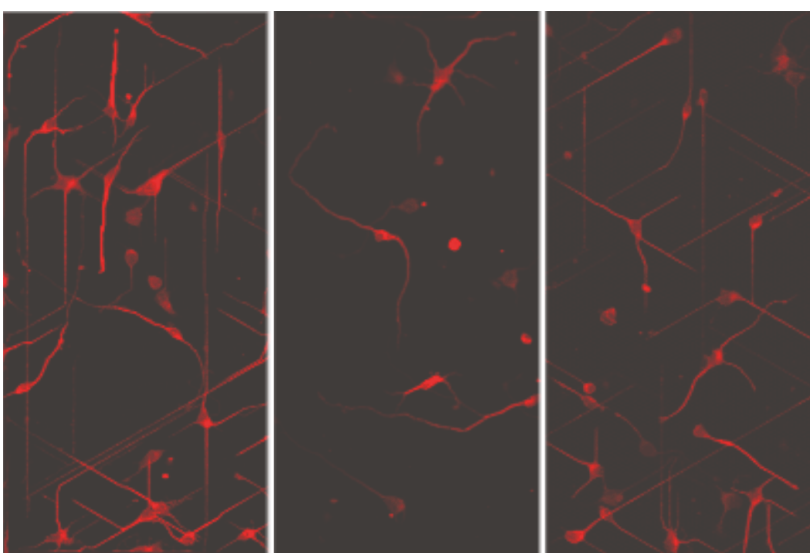

(b)

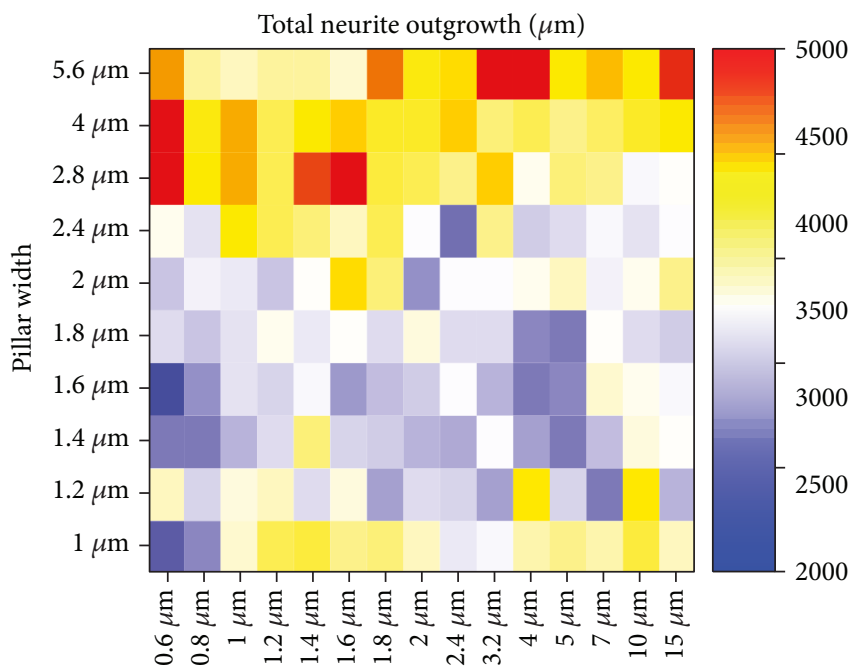

Pillar spacing

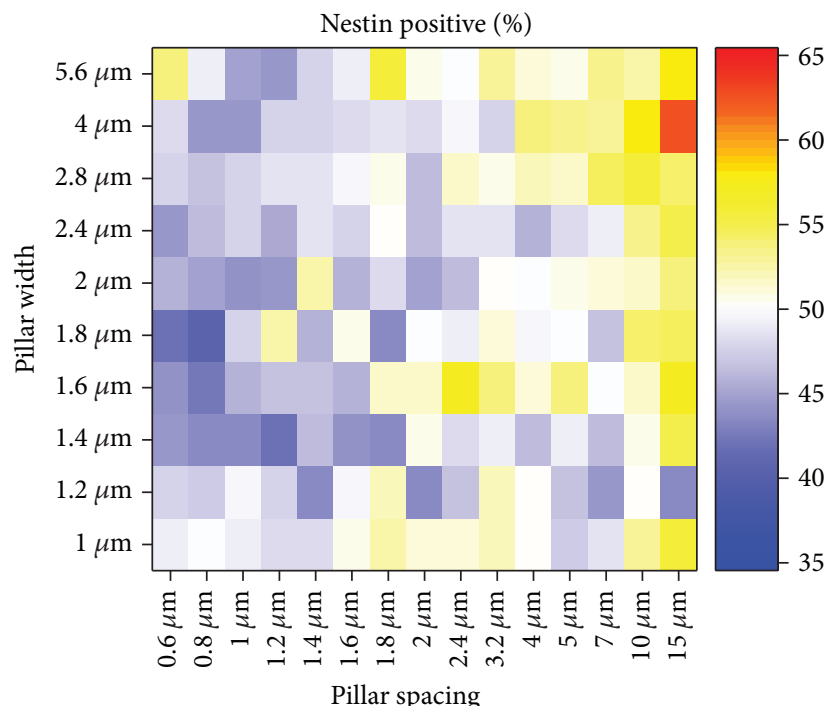

(c)

Figure 2: Analysis of neurite length and cell identity on different pillar topographies. (a) Schematic design of the micropillar substrate according to Micholt et al. Reproduced with the permission of the authors. (b) Examples of neuronal morphology on different pillar configurations (H9 neuronal progeny on pillar configuration: spacing $1.0 \mu \mathrm{m} /$ width $1.2 \mu \mathrm{m}$; spacing $1.8 \mu \mathrm{m} /$ width $5 \mu \mathrm{m}$; spacing $2.8 \mu \mathrm{m} /$ width $1.4 \mu \mathrm{m}$ ). Cells were stained with an antibody against TUJ1. (c) Heatmap plots in function of width and spacing, representing the cell number, total neurite outgrowth, and presence of the neuronal marker TUJ1 and progenitor marker NESTIN, $24 \mathrm{~h}$ after plating. 

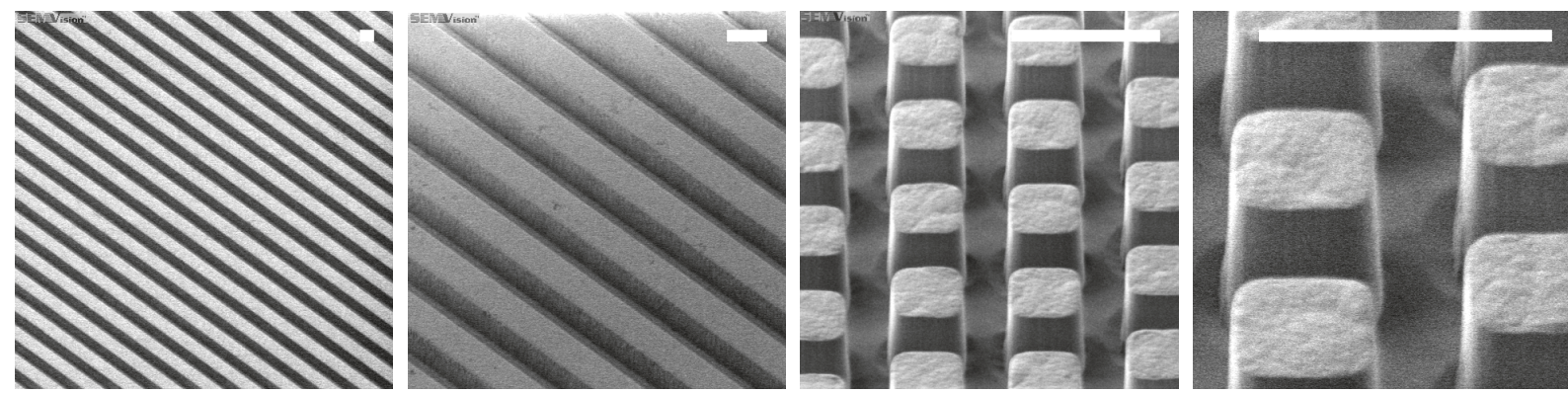

Figure 3: Scanning electron microscopy of the surface topography of microgroove and micropillar silicon oxide substrates. Scale bar represents $5 \mu \mathrm{m}$.
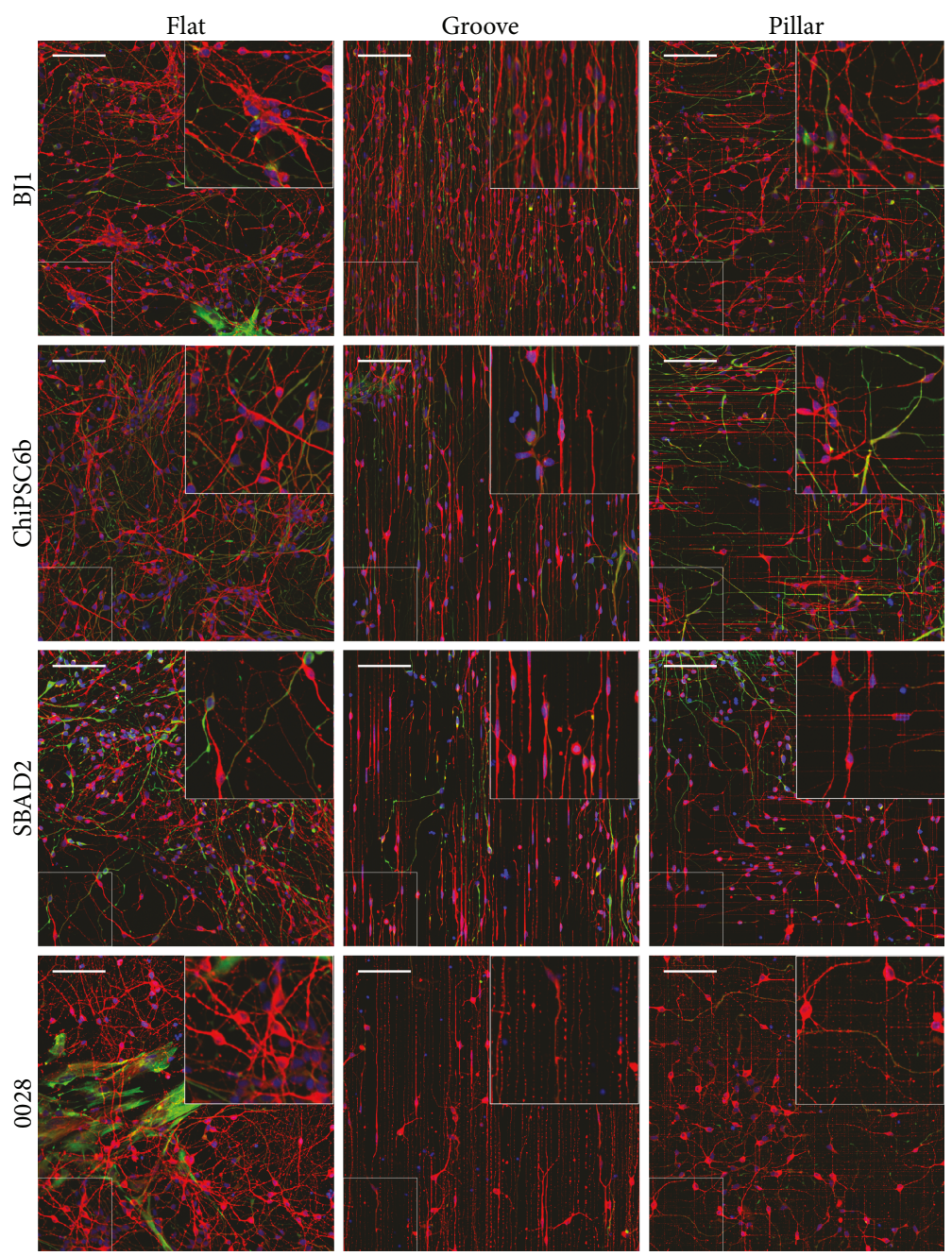

FIGURE 4: Immunofluorescent staining of human PSC-derived cortical neurons, cultured for 15 days on flat, pillar, and grooved topographies. Neurons were stained with Hoechst 33258, and antibodies against the neuronal marker, $\beta 3$-tubulin (TUJ1; red color), and the progenitor marker, NESTIN (green color). Scalebar represents $100 \mu \mathrm{m}$. Picture insets represent a three times magnified detail of the original picture.

3.4. Topography Promotes Neuronal Differentiation. To assess the possible effect of the different topographic surfaces on the differentiation of PSC-derived cortical neurons, we examined the neuronal progeny by semiautomated image analysis (MetaMorph), including quantification of the total cell numbers and presence of TUJ1 positive neurons and
NESTIN positive NPCs. We analysed neuronal progeny from the 4 different PSC lines (three biological replicates for ChiPSC6b, BJ1, and 0028; two biological replicates for the SBAD2 line). Unlike primary neuronal cultures, PSC-derived cortical neuron cultures continue to expand in culture, as a pool of NPCs persists among the differentiating cortical 

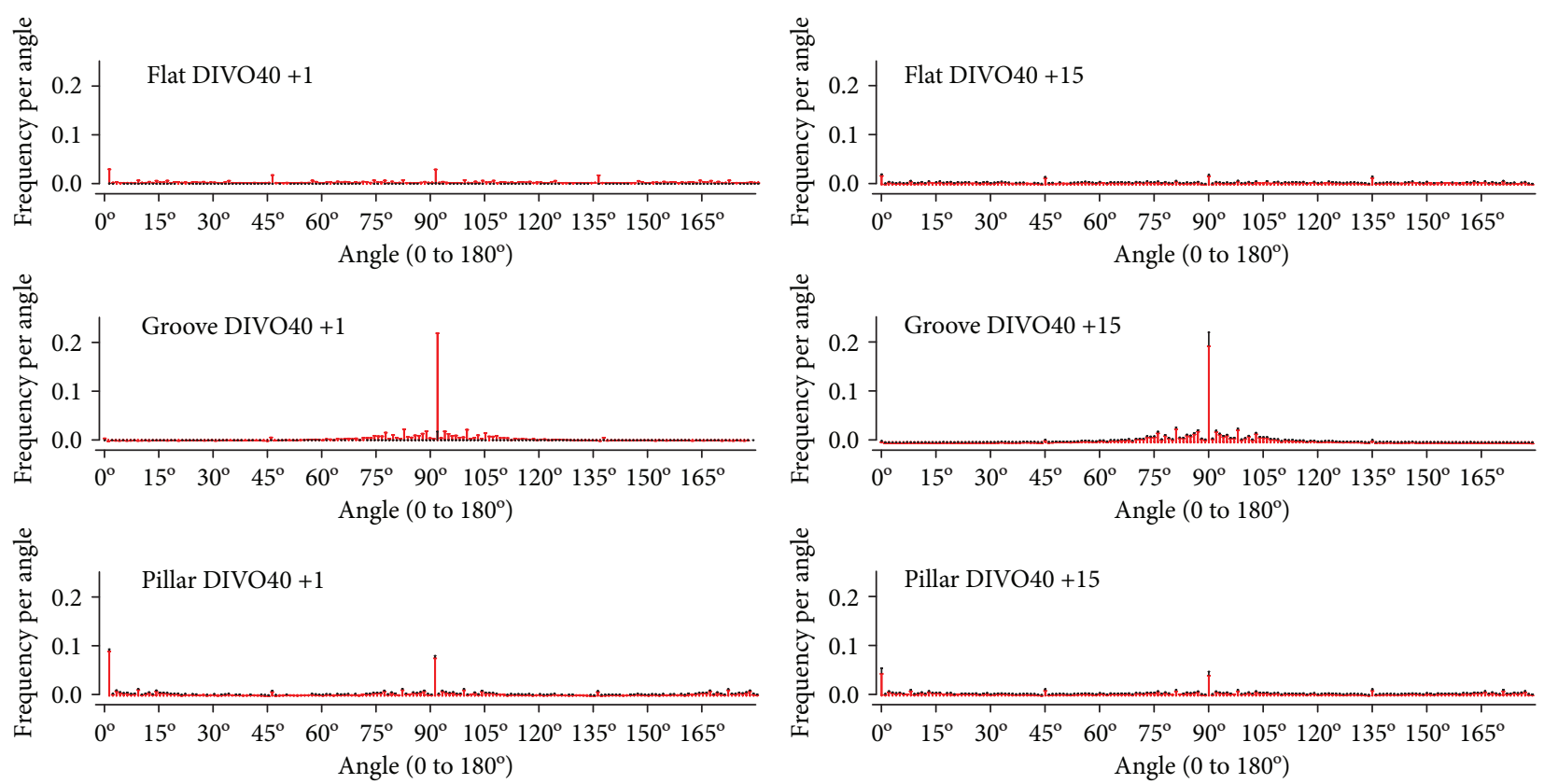

FIgURE 5: Directionality histograms ranging from 0 to $180^{\circ}$. Neuronal alignment was analysed for short-term (24 hour) and long-term (15 days) neuronal cultures. Average directionality measurements for cortical neurons derived from 4 PSC lines (2-3 different differentiations per cell line) are presented in the graph.

neurons. Human cortical neurogenesis is prolonged compared to its mouse counterpart and continues for $>100$ days in vitro [18]. As expected, prolonged culture of PSC-derived cortical cultures increased the total cell number (mean absolute cell number on flat topography: $195 \pm 121$ (DIV40+1) and $655 \pm 385$ (DIV40+15)). However, this increase was significantly lower for PSC progeny cultured on microtopography (Figure 6(a)). Compared to the flat surface topography on DIV $40+15$, the absolute cell number was $417 \pm 232$ cells grown on microgrooves (mean difference 238 cells, $P=$ $\left.0.0001,{ }^{* * *}\right)$ and $425 \pm 191$ cells for cells grown on micropillars (mean difference 230 cells, $P=0.0002,{ }^{* * *}$ ). Morphological analysis and quantification of neurite outgrowth showed that microtopography enhanced neurite outgrowth (Figure 6(b)). The mean neurite outgrowth per cell on DIV $40+15$ was $101.2 \pm 53.9 \mu \mathrm{m}$ for flat surfaces and increased to $173.0 \pm 85.8 \mu \mathrm{m}$ for microgroove substrates (1.71 fold change, $\left.P<0.0001,{ }^{* * * *}\right)$ and $144.7 \pm 62.4 \mu \mathrm{m}$ for micropillar substrates ( 1.43 fold change, $\left.P=0.0152,{ }^{*}\right)$. The number of cells that stained positive for the progenitor marker NESTIN decreased from an average of $53.8 \pm 21.0 \%$ on flat substrates to $34.7 \pm 18.9 \%$ for microgroove substrates and $31.8 \pm 22.6 \%$ for micropillar substrates (mean difference in \%; flat vs. groove: 19.1, $P<0.0001$, ${ }^{* * * *}$; flat vs. pillar: $22.0, P<0.0001$, $\left.{ }^{* * * *}\right)$ (Figure 6(c)), which could be consistent with enhanced differentiation in response to surface topography. Because NPCs also stained weakly with anti-TUJ1 antibodies, the decrease in NESTIN positive cells was not paralleled with an increased frequency of TUJ1 positive cells. Expression of TUJ1 was similarly high on all surfaces (Figure 6(d)), indicating that the differentiating cultures retained their neuronal identity and did not differentiate towards, for example, astrocytes.

\section{Discussion}

We here describe the effect of topographical cues present in silicon oxide substrates, compatible with the construction of MEAs, on the behavior of human pluripotent stem cellderived cortical neurons. We demonstrated that plating of PSC neural progeny on microgroove and micropillar substrates significantly affected the number of differentiating neuronal cells from PSCs, significantly decreased the frequency of immature NESTIN positive NPCs persisting in PSC progeny, and increased the neurite outgrowth length, as well as neurite alignment. We demonstrated similar results for multiple PSC lines (embryonic and iPSC-derived) indicating that the response to topography was cell line independent.

The response of cells to topography has been shown to be dependent on the substrate and the specific surface features that are being introduced, as recently reviewed by Simitzi et al. and Marcus et al. $[19,20]$. However, most studies have addressed the effect of topography on neuronal cell behavior using primary neuronal progenitors. Although some studies assessed the effect of substrate modifications on PSCderived neurons, they were focused on initial stem cell fating and used mostly soft substrates [10-12]. By contrast, we here chiefly assessed the effect of topography on neuronal maturation and neurite outgrowth and directional guidance, as we purified cortical PSC-NPCs (DIV40), such that cell fating to the neuronal lineage was completed at the time of plating on the topographical chips. In addition, rather than using PDMS- or PUA-based substrates, we tested the topography present in silicon oxide-based rigid substrates, with the aim to subsequently convert the chips to MEAs. Therefore, we first confirmed that the silicon-based topographies that were shown to support neuronal guidance of embryonic mouse 


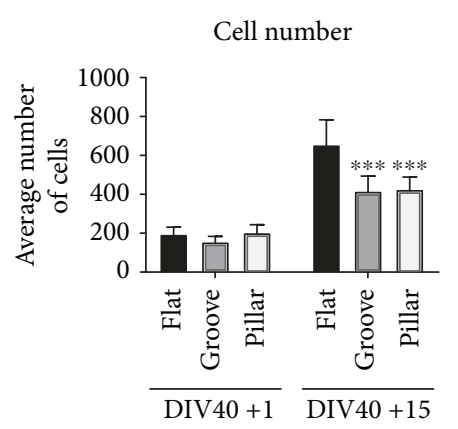

(a)

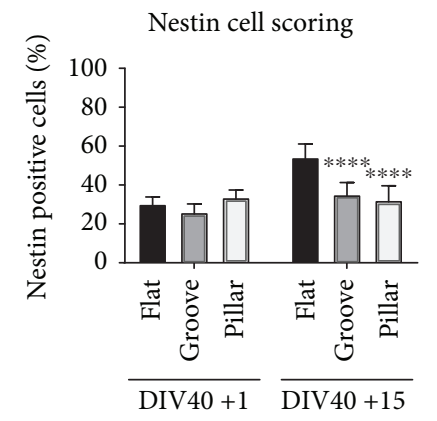

(c)

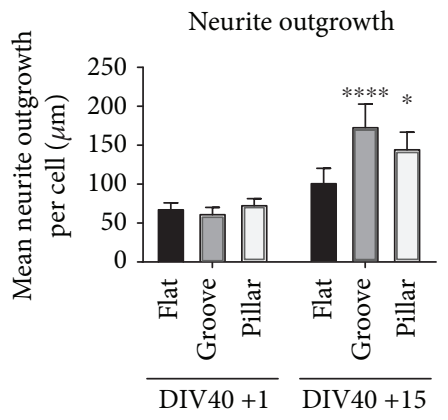

(b)

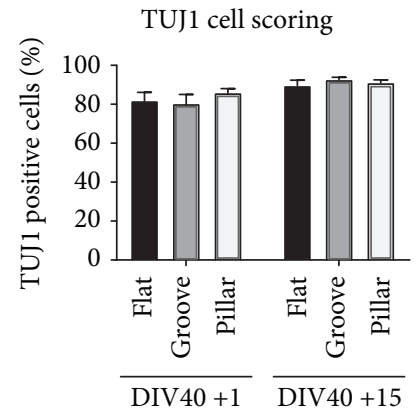

(d)
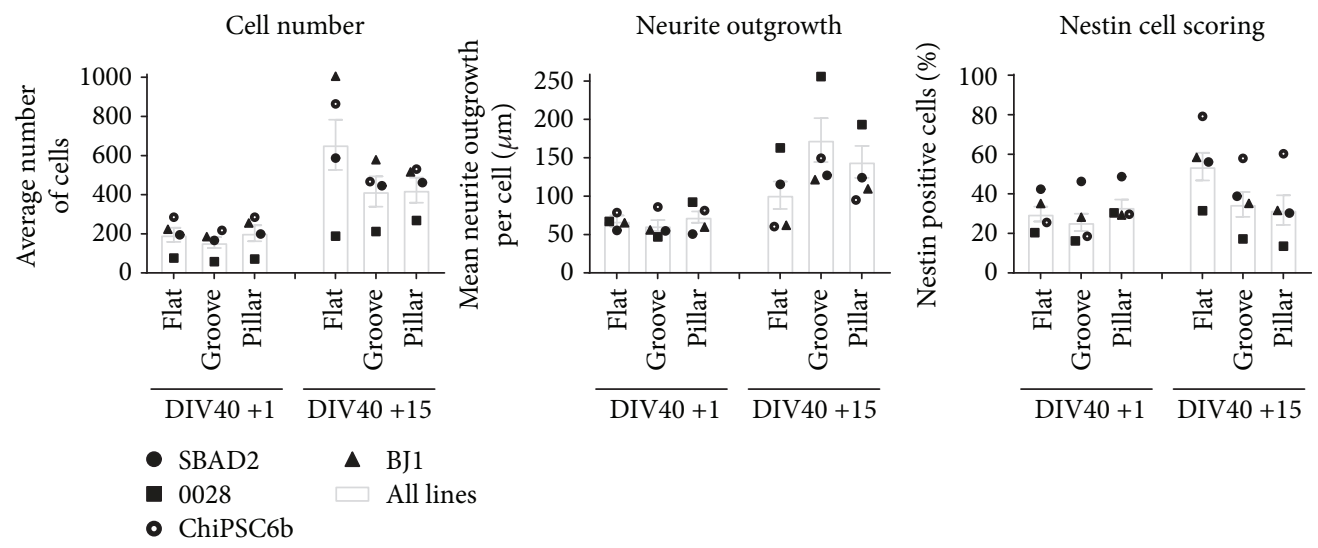

(e)

Figure 6: Cortical neuron differentiation of human PSC on microtopography substrates: (a) quantification of total cell numbers, (b) mean neurite outgrowth per cell, and subpopulations of (c) neuronal progenitors (NESTIN positive cells) and (d) neurons (TUJ1 positive cells). Averaged data of cortical differentiations of the SBAD2, 0028, ChiPSC6b, and BJ1 iPSC lines. (e) Quantification of cell number, average neurite outgrowth, and NESTIN expression. Averaged data for each individual PSC line is plotted on top of the combined data (bar chart). Within each time point, we compared flat, groove, and pillar substrates.

hippocampal neurons (Micholt et al. [13]) also supported neuronal guidance of immature DIV40+1 PSC neuronal progeny. We subsequently designed novel topographical chips, in which pillar morphology was changed to allow easy inclusion of electrodes, and with inclusion of microgroove topographies, also enabling subsequent incorporation of electrodes. These novel topographical chips revealed that guidance of neurites of both immature DIV40+1 progeny, as well as neuronal cells with a lower cortical layer phenotype (DIV40+15), could be induced at different angles depending on the topography. Additional experiments will be needed to confirm whether peripheral nervous system neurons (e.g., spinal motor neurons or peripheral sensory neurons) respond in a similar fashion to surface topography.

It is well known that persistence of NPCs among differentiating cortical neurons, as demonstrated by immunofluorescence (NESTIN expression) and qRT-PCR (PAX6 transcript levels), complicates long-term cultivation of hPSC-derived cortical neurons. Continued expansion of progenitors can lead to culture overgrowth, and this often negatively affects experiments, especially in the context of electrophysiological assessments. Introducing substrate topography can either expand the growth area (shallow features) or limit the growth area (high topographical features), which are believed to 
potentially influence cell proliferation and/or differentiation/ maturation. We observed that significantly fewer cells were present on DIV40+5, when cells were cultured on microgroove or micropillar substrates. Song et al. previously demonstrated lower 5-bromo-2-deoxyuridine (BrdU) incorporation when PSCs were differentiated on poly(dimethylsiloxane) topographical substrates, which may suggest that in the current studies, NPC proliferation is inhibited by topographical features [11]. However, as the stiffness and elasticity of poly(dimethylsiloxane) substrates differ significantly from the silicon oxide chips used in the current study, we cannot simply extrapolate the finding by Song et al. to our studies. Therefore, further studies deciphering the contribution of cell death vs. cell proliferation to the lower neuron number on DIV40+15 will be required. Concomitantly with the observed reduction of the NPC pool on microgrooves and micropillars, we could anticipate to see a reduction in the number of astrocytes in longer-term cultures. However, as human cortical neurogenesis is lengthy, with the switch from neurogenesis to gliogenesis occurring around 60 days in vitro [3], cultures up to 100 days in vitro would be required to confidently assess the effect of topography on spontaneous gliogenesis.

In addition, to start to assess if topographical chips aid in neuronal maturation, we quantified neurite outgrowth as well as persistence of NESTIN positive immature NPCs in DIV40+15 progeny on flat vs. nanogroove vs. nanopillar surfaces. We demonstrated that progeny on pillars and grooves displayed more extensive neurite outgrowth and contained fewer NESTIN positive cells. However, additional studies on cells maintained for longer periods of time on the topographical surfaces will be required to prove that these topographies induce neuronal maturation. Furthermore, once electrodes have been incorporated in the chips, we will be able to follow neuronal maturation based on the creation of functional synaptic neuronal networks in response to the different topographies. Under current conventional culture conditions, human PSC-derived neuronal cells require on average more than two months of in vitro culture before functional synapses are formed [3,4]. It will be of interest to test if topographical surfaces can decrease the time required for generating neurons with mature features. Furthermore, the neurite guidance conferred by the topography should greatly enhance the assessment of action potential propagation along the neuronal axon; therefore, neurite guidance by means of surface topography may provide novel opportunities in the electrophysiological characterization of neurons.

\section{Conclusions}

We investigated the response of human iPSC- and ESCderived cortical neurons to substrate topography. Topography induced guidance, improved neurite outgrowth, decreased the number of immature NPCs, and decreased proliferation. Thus, we hypothesize that embedding topography in silicon substrates is an effective approach to direct and improve neuronal network formation. Based on these results, we propose to integrate the topographies identified here in multielectrode arrays as a practical method to control the neuro-electronic interface. The neurite guidance conferred by the topography should greatly enhance the assessment of action potential propagation along the neuronal axon. Finally, the embedding of topography in silicon substrates is compatible with the concomitant miniaturisation of culture chambers for high-throughput applications.

\section{Data Availability}

The datasets generated and/or analysed during the current study are available from the corresponding author on reasonable request.

\section{Disclosure}

IMEC is a nonprofit research institute with no commercial activities.

\section{Conflicts of Interest}

The authors declare that there is no conflict of interest regarding the publication of this paper.

\section{Funding}

This research was supported by the Agency for Innovation by Science and Technology, Flanders (IWT SBO iPSCAF-150031 and IWT-VIND project-135043). JT is a recipient of the IWT SB (141228). PVD holds a senior clinical investigatorship of FWO-Vlaanderen and is supported by the ALS Liga Belgium and the KU Leuven ALS funds "Een hart voor ALS" and "Laeversfonds voor ALS onderzoek."

\section{Supplementary Materials}

Table S1 lists the primer sequences used in this study. Figure S1 depicts the isotype controlled immunofluorescent staining of the primary antibodies used in this study. (Supplementary Materials)

\section{References}

[1] K. Takahashi and S. Yamanaka, "Induction of pluripotent stem cells from mouse embryonic and adult fibroblast cultures by defined factors," Cell, vol. 126, no. 4, pp. 663-676, 2006.

[2] K. Okita, T. Ichisaka, and S. Yamanaka, "Generation of germline-competent induced pluripotent stem cells," Nature, vol. 448, no. 7151, pp. 313-317, 2007.

[3] Y. Shi, P. Kirwan, J. Smith, H. P. C. Robinson, and F. J. Livesey, "Human cerebral cortex development from pluripotent stem cells to functional excitatory synapses," Nature Neuroscience, vol. 15, no. 3, pp. 477-486, 2012.

[4] I. Espuny-Camacho, K. A. Michelsen, D. Gall et al., "Pyramidal neurons derived from human pluripotent stem cells integrate efficiently into mouse brain circuits in vivo," Neuron, vol. 77, no. 3, pp. 440-456, 2013.

[5] M. R. Livesey, D. Magnani, G. E. Hardingham, S. Chandran, and D. J. A. Wyllie, "Functional properties of in vitro excitatory cortical neurons derived from human 
pluripotent stem cells," The Journal Physiology, vol. 594, no. 22, pp. 6573-6582, 2015.

[6] M. K. Lewandowska, D. J. Bakkum, S. B. Rompani, and A. Hierlemann, "Recording large extracellular spikes in microchannels along many axonal sites from individual neurons," PLoS One, vol. 10, no. 3, article e0118514, 2015.

[7] A. Ferrari, M. Cecchini, M. Serresi, P. Faraci, D. Pisignano, and F. Beltram, "Neuronal polarity selection by topographyinduced focal adhesion control," Biomaterials, vol. 31, no. 17, pp. 4682-4694, 2010.

[8] R. Palankar, M. Glaubitz, U. Martens et al., "3D micropillars guide the mechanobiology of human induced pluripotent stem cell-derived cardiomyocytes," Advanced Healthcare Materials, vol. 5, no. 3, pp. 335-341, 2015.

[9] A. Reimer, A. Vasilevich, F. Hulshof et al., "Scalable topographies to support proliferation and Oct4 expression by human induced pluripotent stem cells," Scientific Reports, vol. 6, no. 1, article 18948, 2016.

[10] M. R. Lee, K. W. Kwon, H. Jung et al., "Direct differentiation of human embryonic stem cells into selective neurons on nanoscale ridge/groove pattern arrays," Biomaterials, vol. 31, no. 15, pp. 4360-4366, 2010.

[11] L. Song, K. Wang, Y. Li, and Y. Yang, "Nanotopography promoted neuronal differentiation of human induced pluripotent stem cells," Colloids and Surfaces B: Biointerfaces, vol. 148, no. 148, pp. 49-58, 2016.

[12] L. Y. Chan, W. R. Birch, E. K. F. Yim, and A. B. H. Choo, "Temporal application of topography to increase the rate of neural differentiation from human pluripotent stem cells," Biomaterials, vol. 34, no. 2, pp. 382-392, 2013.

[13] L. Micholt, A. Gärtner, D. Prodanov, D. Braeken, C. G. Dotti, and C. Bartic, "Substrate topography determines neuronal polarization and growth in vitro," PLoS One, vol. 8, no. 6, article e66170, 2013.

[14] S. Yamanaka, "Induced pluripotent stem cells: past, present, and future," Cell Stem Cell, vol. 10, no. 6, pp. 678-684, 2012.

[15] S. Raitano, L. Ordovas, L. De Muynck et al., "Restoration of progranulin expression rescues cortical neuron generation in an induced pluripotent stem cell model of frontotemporal dementia," Stem Cell Reports, vol. 4, no. 1, pp. 16-24, 2015.

[16] J. A. Thomson, J. Itskovitz-Eldor, S. S. Shapiro et al., "Embryonic stem cell lines derived from human blastocysts," Science, vol. 282, no. 5391, pp. 1145-1147, 1998.

[17] A. Rajnicek, S. Britland, and C. McCaig, "Contact guidance of CNS neurites on grooved quartz: influence of groove dimensions, neuronal age and cell type," Journal of Cell Science, vol. 110, Part 23, pp. 2905-2913, 1998.

[18] N. Gaspard, T. Bouschet, R. Hourez et al., "An intrinsic mechanism of corticogenesis from embryonic stem cells," Nature, vol. 455, no. 7211, pp. 351-357, 2008.

[19] M. Marcus, K. Baranes, M. Park, I. S. Choi, K. Kang, and O. Shefi, "Interactions of neurons with physical environments," Advanced Healthcare Materials, vol. 6, no. 15, article 1700267, 2017.

[20] C. Simitzi, A. Ranella, and E. Stratakis, "Controlling the morphology and outgrowth of nerve and neuroglial cells: the effect of surface topography," Acta Biomaterialia, vol. 51, pp. 21-52, 2017. 


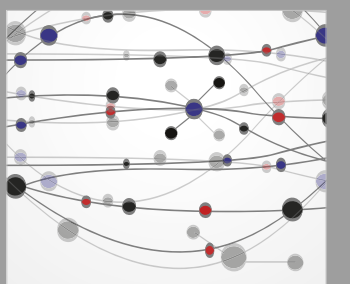

The Scientific World Journal
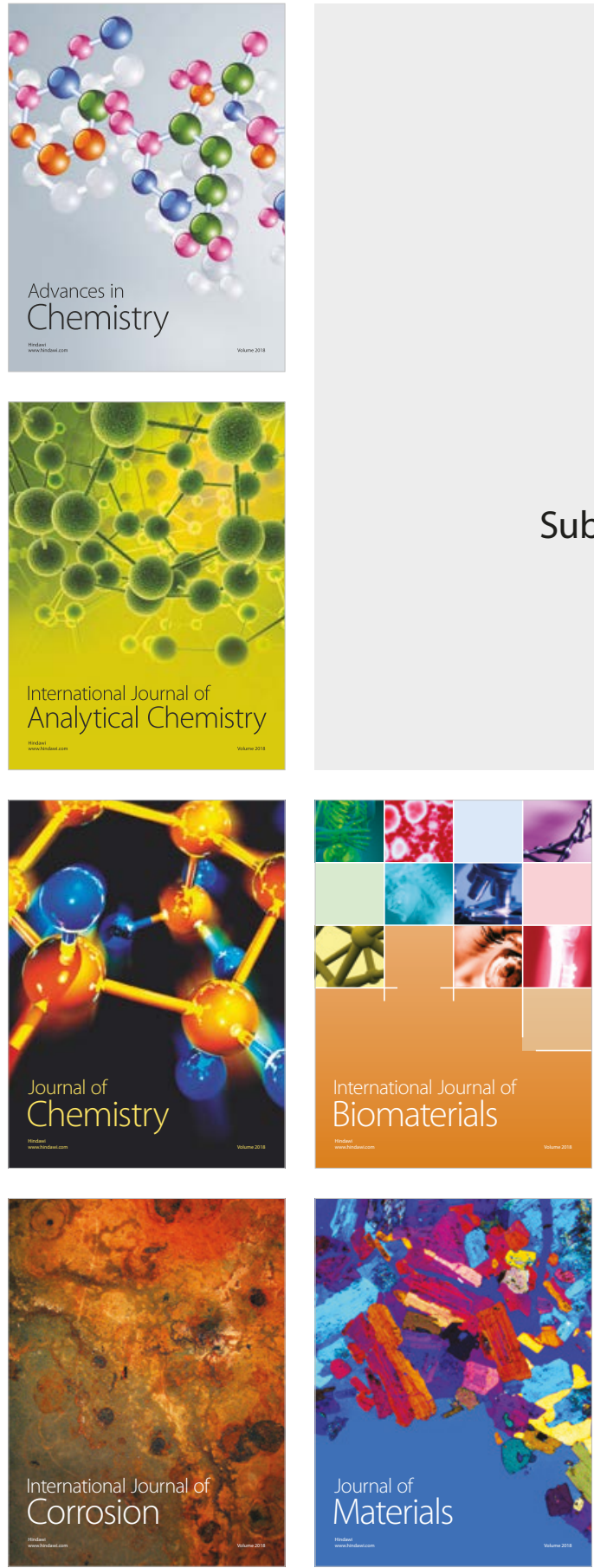

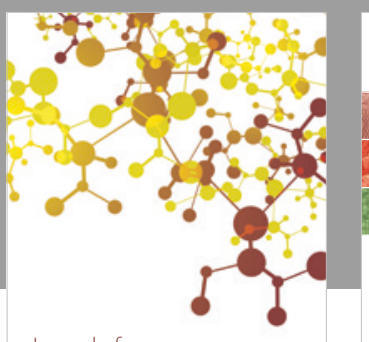

Journal of

Applied Chemistry
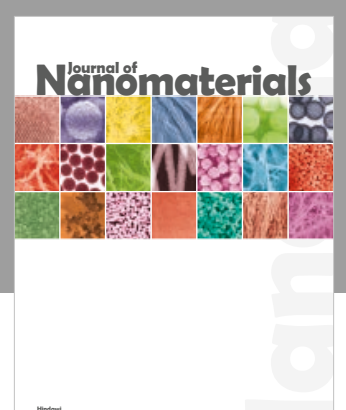

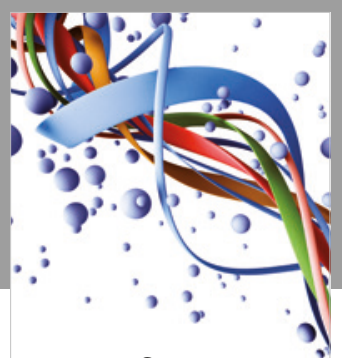

Scientifica

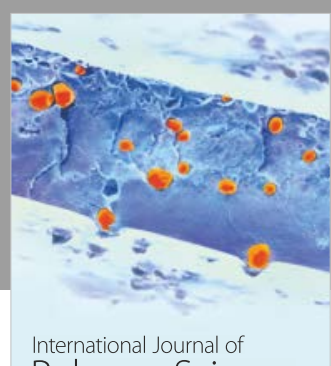

Polymer Science

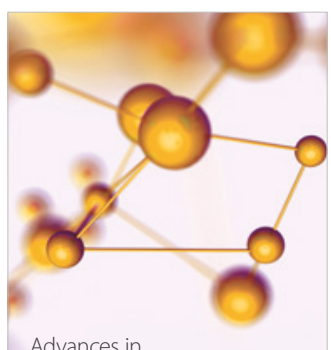

Physical Chemistry
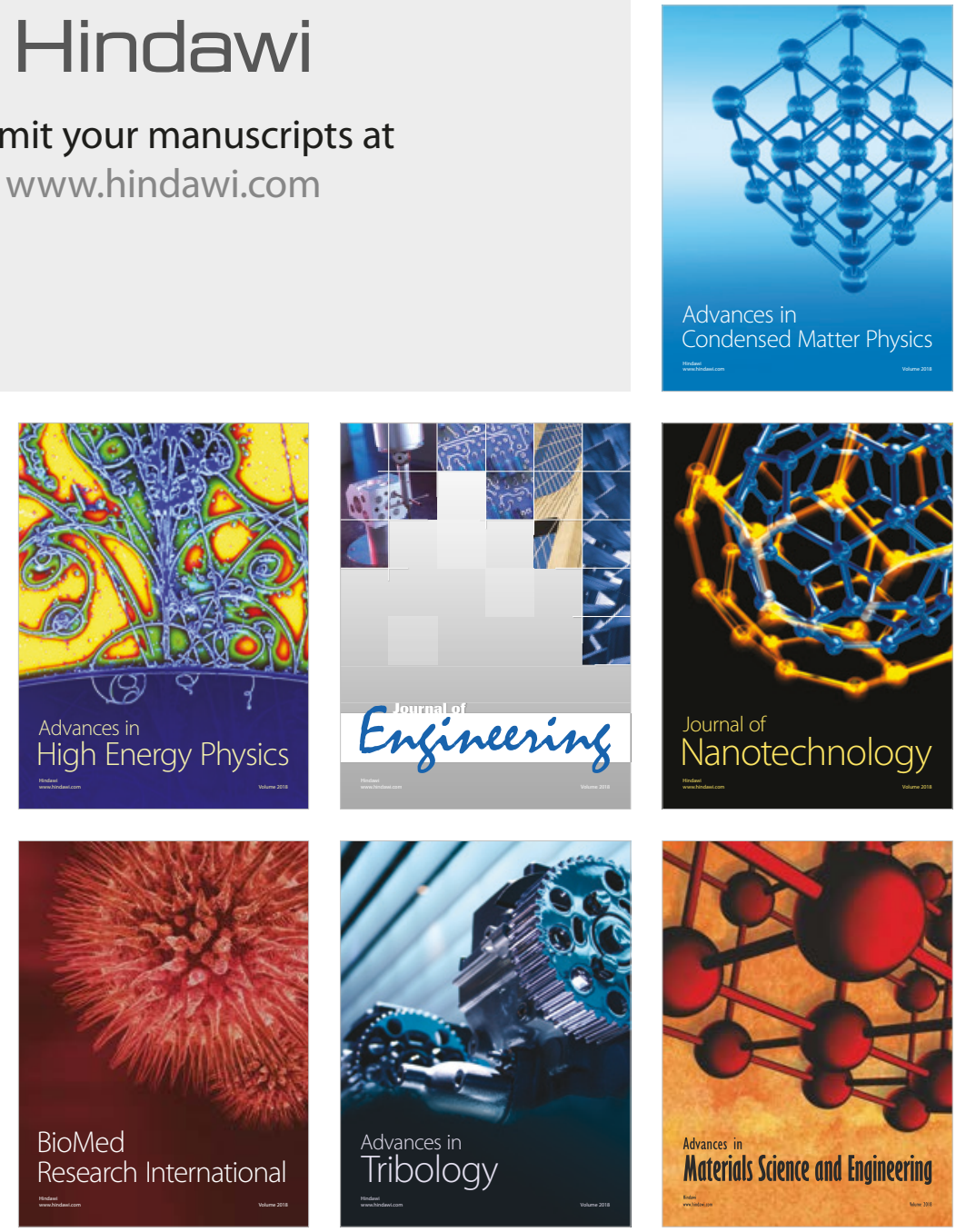